\title{
Multilinguales
}

$14 \mid 2020$

Enseignement universitaire et professionnalisation : enjeux, questionnements et défis socio-économiques

\section{Pratiques langagières et glottophobie dans la ville de Bejaia : quand la langue est au service de la discrimination}

Language practices and glottophobia in the city of Bejaia: when language is at the service of discrimination

\section{Lydia Benbelaid}

\section{(2) OpenEdition}

\section{Édition électronique}

URL : https://journals.openedition.org/multilinguales/5768

DOI : 10.4000/multilinguales. 5768

ISSN : 2335-1853

\section{Éditeur}

Université Abderrahmane Mira - Bejaia

\section{Référence électronique}

Lydia Benbelaid, «Pratiques langagières et glottophobie dans la ville de Bejaia : quand la langue est au service de la discrimination », Multilinguales [En ligne], 14 | 2020, mis en ligne le 10 décembre 2020, consulté le 02 juillet 2021. URL : http://journals.openedition.org/multilinguales/5768 ; DOI : https:// doi.org/10.4000/multilinguales.5768

Ce document a été généré automatiquement le 2 juillet 2021. 


\title{
Pratiques langagières et glottophobie dans la ville de Bejaia : quand la langue est au service de la discrimination
}

\author{
Language practices and glottophobia in the city of Bejaia: when language is at \\ the service of discrimination
}

Lydia Benbelaid

L'étude des langues en milieu plurilingue est toujours très complexe car, du fait de se partager un même espace géographique, les langues sont en perpétuelle concurrence pour se maintenir. À Bejaia, comme dans la plupart des villes algériennes, la question linguistique est très complexe car en plus de ses langues, cette ville possède aussi un parler propre à elle appelé communément le bougiote (De Habsbourg, 1999), qui est tantôt vu comme un arabe kabylisé, et tantôt comme un kabyle arabisé (Benbelaid, 2014). Ce parler typique de cette région est devenu plus qu'un moyen de communication, il est le principal élément définitoire de cette ville. D'ailleurs, on attribue ce parler à ce qu'on désigne comme les grandes familles de Bejaia (Aoumer, 2009) et qui n'ont rien avoir avec les nouveaux habitants. Aussi, cette façon d'associer des origines à la langue a fini par engendrer des discriminations et des comportements dits " glottophobes ».

2 Cette contribution s'intéresse à cet univers linguistique très particulier, et a pour objectif de lever le voile sur le phénomène de glottophobie dans les milieux plurilingues. Elle s'inscrit plus particulièrement dans une tendance théorique qui vise à saisir les tensions sociales, les faits de ségrégation, et la mise en mots des catégories de la discrimination (Bulot, 2008). Elle pose comme principal postulat que l'attachement au parler bougiote a fini par faire naître des jugements à l'égard des autres parlers, et qu'il existe une discrimination selon les profils langagiers. La réflexion proposée ici se centre particulièrement sur la perception et la catégorisation des différents parlers vus comme stimulus des comportements glottophobes dans cette ville. En effet, la plupart des recherches sur la glottophobie préfèrent mettre l'accent sur ceux qui subissent les 
comportements glottophobes, et rarement sur ceux qui les font subir. Hors, comprendre les raisons qui poussent certains individus à avoir un comportement glottophobe pourrait permettre de mieux comprendre ce phénomène. Nous souhaitons ainsi savoir: quel est le degré d'implantation des comportements glottophobes? Et qu'est-ce qui les motive?

\section{Retour sur le concept de glottophobie}

3 Parler de glottophobie est assez complexe, car comme toutes les formes de discrimination, elle n'est jamais vraiment assumée par les individus. Le phénomène de discrimination langagière est très présent en milieu plurilingue où les langues n'ont pas toutes le même statut et où elles subissent des évaluations constantes qui modifient le rapport des individus à leur égard et, de ce fait, aucun rapport aux langues n'est complètement neutre. Les jugements négatifs à l'égard de certains parlers sont de plus en plus fréquents et ont un impact direct sur le rapport à l'autre (Boutet, 1997).

4 En général, le terme glottophobie est utilisé pour désigner une discrimination linguistique, il indique une ségrégation basée exclusivement sur la langue. Selon Blanchet (2016), la glottophobie est un terme qui englobe tout mépris, haine, agression, rejet, ou exclusion d'une personne sur le simple fait de considérer incorrectes, inférieures, ou mauvaises certaines formes linguistiques employées par cette personne. Ce terme composé de Glotto qui signifie « langue » en grec, et phobie qui renvoie à «la peur ", est utilisé pour définir donc les cas de discrimination et de dévalorisation linguistique, déconsidérant ainsi certaines formes linguistiques. Le concept de glottophobie englobe les discriminations linguistiques et les stigmatisations et jugements négatifs qu'une personne peut subir uniquement parce que son parler est vu comme inférieur. Ainsi, selon Blanchet: "appliquée à des pratiques linguistiques autres, stigmatisées en elles-mêmes, pour elles-mêmes, et avec elles les personnes et les groupes qui les parlent et qui s'y identifient, personnes et groupes dont les capacités humaines au langage sont dès lors réputées sous développées » (Arditty \& Blanchet : 2008).

Bien que ces discriminations et inégalités ne soient jamais ouvertement admises, elles n'en demeurent pas moins très visibles et cachent des enjeux sociaux beaucoup plus importants. Nous reprenons ici ces quelques mots d'Arditty \& Blanchet (2008) qui résument un peu ce phénomène :

Chaque variante linguistique est dotée socialement d'une valeur symbolique différente [...] et la "légitimité » à la produire qui est reconnue à celui qui la produit, ceci étant intriqué dans des processus de domination, d'hégémonie et de conflits. Ces hiérarchisations et les valeurs symboliques qui y sont liées, ne sont pas attachées "par nature» aux formes linguistiques elles-mêmes, toutes potentiellement dominantes et valorisées. Ces hiérarchisations proviennent des structures sociales qu'elles contribuent à produire et à reproduire.

Bien que cette vision soit assez extrême, nous pensons qu'il existe des formes de glottophobie plus subtiles. En effet, certaines discriminations peuvent se manifester dans des situations sans lien direct avec des différences ethniques, religieuses, ou culturelles (Chachou, 2012); il semblerait que dans certains cas les individus d'une même origine voire d'une même région peuvent être confrontés au phénomène de glottophobie (Blanchet, 2018). 


\section{Corpus et méthodologie}

6 L'objectif de cette recherche était de comprendre comment sont perçues les personnes issues de milieux linguistiques différents. L'enquête par questionnaire a été réalisée sur une tranche d'âge assez jeune. En effet, nous nous sommes tournée vers une centaine d'étudiants résidants dans la ville de Bejaia pour effectuer notre enquête car, pour nous, cette tranche de la population est la plus consciente des phénomènes liés aux pratiques langagières et au rapport entre locuteurs. Nos informateurs ont été amenés à aborder plusieurs questions relatives aux représentations, à l'identité linguistique, et aux pratiques discursives. Ce qui a, d'ailleurs, clairement laissé paraître des discriminations langagières dont nous débattrons plus en détail par la suite.

Nous avons organisé notre méthodologie pour répondre à des objectifs bien précis que nous pouvons résumer comme suit :

- Comprendre l'importance et le rôle de langue maternelle dans le rapport à l'autre ;

- Cerner l'importance de l'identité linguistique ;

- Mesurer le degré de glottophobie à travers le rapport à l'espace ;

- Saisir le rôle des représentations dans le phénomène de glottophobie.

\section{Autour de la langue maternelle et des frontières linguistiques}

7 Il est communément admis que ce qui détermine l'importance d'une langue ce n'est pas seulement son statut mais aussi son usage et son importance aux yeux des locuteurs. Vénéré par tous les habitants de la ville de Bejaia, le bougiote est très présent dans leurs pratiques quotidiennes. Ce parler est très différent de ceux des autres régions du fait de son long contact avec le kabyle qui lui a donné toute sa spécificité, et l'exemple le plus fréquemment donné pour illustrer cette différence est : baba dayen ğa $=$ «Papa aussi est venu» :

- baba : de l'arabe qui signifie 'papa' (en kabyle : vava)

- dayen : du kabyle qui signifie 'aussi'

- ğa : de l'arabe qui signifie 'venu'- il est venu (en kabyle : youssade)

Toutefois, ces dernières années il ne domine plus autant à Bejaia et se voit concurrencé chaque jour un peu plus par le kabyle. En effet, il suffit d'un petit tour dans la ville pour se rendre compte que l'arabe bougiote doit composer avec le kabyle sur son propre territoire (Aoumer, 2009).

Aussi, nos premières questions étaient toutes centrées sur la langue maternelle, son usage et son importance pour nos informateurs, car les pratiques discursives sont une sorte de miroir qui reflète les tensions et les pressions présentes dans la société. Ainsi, nous avons pu constater qu'au coté de l'arabe bougiote une forte présence du kabyle ${ }^{1}$ qui semble ainsi gagner de plus en plus de terrain. En effet, $68 \%$ de nos enquêtés disent avoir le kabyle comme langue maternelle, un taux assez important qui prouve bien que le kabyle tend à concurrencer, voire à devancer le bougiote. Ainsi, même si le bougiote a dominé dans la ville de Bejaia, l'exode rural qu'elle a connu ces dernières années tend à modifier cette donne et à le faire régresser. Et c'est, sans doute, ce qui explique cette méfiance des bougiotes à l'égard de ceux qu'ils surnomment les arrivistes² 
9 En effet, ces nouveaux arrivants sont considérés par les bougiotes comme des étrangers et des montagnards qui ne connaissent rien à la vie des citadins. Et ils voient en eux une menace pour leur langue maternelle et, par la même, pour leur identité, nous reprenons ici ces quelques mots d'Ibtissem Chachou (2019) pour expliquer cela : «les anciens citadins se considèrent comme les habitants légitimes des lieux, alors que les ruraux seraient d'indus occupants, voire des "envahisseurs " [...] Les ruraux, considérés comme un corps étranger ». Cette façon de voir l'autre en dit long sur l'importance que représente la langue maternelle pour ses usagers. En effet, le sentiment qu'un jour le kabyle pourrait supplanter le bougiote pourrait justifier ce mépris qu'ont les bougiotes à l'égard de ceux qu'ils nomment les arrivistes.

Plus le temps passe plus il semble évident que le fossé entre les natifs de cette ville et les arrivistes se creuse davantage. Cette barrière qui n'était visible que dans ce que l'on nomme une frontières intra-urbaine (Bulot, 2013), avec le découpage géographique de la ville en haute ville (où vivent les bougiotes) et la plaine $e^{3}$ (occupée par les nouveaux arrivants), semble se transformer en une véritable rivalité où en plus des enjeux linguistiques, il y est aussi question de légitimité.

Ce n'est pas ce qu'il est mais ce qu'il représente qui est important pour les bougiotes, car il renvoie pour eux à la nativité. Il faut dire qu'on attribue ce parler typique des bougiotes qu'aux anciennes familles de Bougie (auxquelles on attribue des origines turques ou même andalouses), et donc aux vrais citadins de cette ville. Toutefois, avec ces nouveaux arrivants, la langue maternelle n'est plus forcement le bougiote, certains privilégient le kabyle parlé par leurs parents. Selon Leila Messaoudi (2001), les ruraux qui s'installent en ville n'abandonnent pas complètement leurs traits linguistiques et cette nouvelle donne fait que les bougiotes tendent à se sentir menacés.

11 L'usage d'une langue dépend exclusivement du choix du locuteur (Lasagabaster, 2006), et certains choix peuvent faire naître des comportements glottophobes. Ainsi, les récents mouvements ruraux qui ont fait agrandir les frontières de la ville, ont aussi contribué à mettre en place un nouveau schéma linguistique où le bougiote n'a plus l'exclusivité et où il doit composer avec le kabyle sur son propre territoire, entraînant ainsi une discrimination des nouveaux venus par les bougiotes qui se confinent, de plus en plus, dans les quartiers de l'ancienne ville comme : le quartier de Bab Llouz, Lhouma Karamane, etc. En effet, selon nos informateurs, les seuls quartiers où le bougiote est très parlé sont ceux qui se situent dans l'ancienne ville uniquement.

Les ruraux sont ainsi vus comme une menace à la langue maternelle qui, au-delà du simple moyen de communication, est ce qui garantit l'unicité du groupe. Le sentiment glottophobe vient principalement de cette envie de protéger et de sauvegarder la langue qui est perçue comme une propriété peu ou pas maitrisée par l'autre. Un héritage des ancêtres qui renvoie à l'identité même de l'individu pour qui il est impossible d'exprimer son identité dans une langue autre que sa langue maternelle. Ces dynamiques identitaires (Bulot, 2004) sous-tendent souvent les tensions intra-urbaines, et nous pouvons ainsi dire que la langue maternelle n'est pas juste une simple composante de l'identité, mais elle contribue à la construction de cette dernière et influe sur le rapport entre les locuteurs. 


\section{Identité linguistique et rapport aux langues}

12 La langue est inextricablement liée à l'identité (Gauthier, 2011), elle est l'un des fondements identitaires les plus importants. D'ailleurs, les recherches sur l'identité durant ces dernières années se révèlent de plus en plus liées aux recherches sur les pratiques langagières, particulièrement en milieu plurilingue. Louis-Jean Calvet (1994) affirme que la réalité des terrains plurilingues nous permet de les envisager comme des lieux de coexistence et de métissage linguistique où se confrontent les différences individuelles, et ces confrontations cachent généralement des enjeux identitaires. Ainsi, quand nous avons demandé à nos informateurs de nous définir ce qu'est l'identité, les réponses nous ont permis de voir à quel point la langue est indéfectiblement liée à l'identité, et à la construction identitaire. Comme nous le confie l'enquêtée $\mathrm{N}^{\circ} 43$ l'identité : «c'est tout les traits qui aident à identifier une personne surtout la langue", et pour l'enquêtée $\mathrm{N}^{\circ} 19$ : "c'est l'ensemble des comportements que possède, s'approprie une personne à savoir: sa langue, ses habitudes, ses traditions, etc.», et pour l'enquêtée $\mathrm{N}^{\circ} 07$ «c'est tout ce qui englobe le mode de vie, la langue ». Ainsi, peu importe la définition que l'on donne à l'identité, la langue y est une composante incontournable. Certains de nos informateurs vont plus loin et nous confient ne pas aimer parler d'autres langues à moins d'y être contraints car cela : «peut entrainer l'oublie de la langue identitaire» [enquêté $\mathrm{N}^{\circ} 69$ ]. Et l'enquêtée $\mathrm{N}^{\circ} 13$ nous affirme, pour sa part, qu'en parlant une autre langue : «la personne pert son identité».

Ces réponses nous permettent de voir que la problématique identitaire se retrouve étroitement liée aux attitudes des locuteurs à l'égard des langues présentes dans leur univers plurilingue (Arezki, 2008), ce qui entraine inexorablement des hiérarchisations et des conflits linguistiques. C'est ce que nous explique Gauthier qui pense que « l'ancrage de l'identité dans la langue, si commun et banalisé qu'il est souvent conçu comme allant de soi [...] on l'associe facilement à une exigence de reconnaissance, jugée légitime dans un certain nombre de cas, elle serait aussi, voire principalement, synonyme d'enfermement, de repli identitaire.» (2011:184). En effet, cette vision restreinte du lien entre langue et identité, en plus d'accentuer le sentiment d'exclusion de certains, ne fait que creuser davantage le fossé qui sépare les habitants de cette ville. Et, à long terme, fait naitre ce qu'on appelle un « repli » identitaire (Thiébault, 2006). D'ailleurs, certains de nos informateurs voient ceux qui ne parlent pas le bougiote comme étant étranger à la ville, voire : «des gens qui n'ont pas de personnalité » [enquêté $\mathrm{N}^{\circ} 47$.

14 La prise de conscience des locuteurs de langues présentées comme « dominantes » fait naitre des hiérarchisations des langues et, par la même, des locuteurs qui les parlent. Ces procédés d'auto-catégorisation et d'hétéro-catégorisation ${ }^{4}$ participent à l'assignation identitaire en société (Chachou, 2019), ce qui entraine inévitablement des tensions, voire des fractures au sein des communautés de locuteurs partageant un même espace. Et c'est justement ce qui est en train de se passer dans la ville de Bejaia.

L'assignation d'une identité en passant par la langue, en plus d'avoir séparé la ville en deux, commence à faire naitre des comportements glottophobes pleinement assumés par certains habitants. Car d'un point de vue sociolinguistique, la construction identitaire passe par le biais d'une "conscientisation" de son appartenance à une même communauté linguistique (Arezki, 2008), et quand certains locuteurs préfèrent ne pas abandonner leur langue maternelle pour le bougiote, on considère qu'ils ne font 
aucun effort d'intégration linguistique. Ainsi, ils sont plus enclins à subir les comportements glottophobes qui sont généralement justifiés par le fait que c'est l'autre qui refuse de s'intégrer. Nous pouvons ainsi dire que la cohabitation entre « les natifs » et « les arrivistes » donne à voir des différences face au rapport aux langues, mais aussi face à leurs utilisations.

\section{Discrimination spatio-langagière}

Dans l'univers sociolinguistique algérien, il y a toujours une forte stigmatisation langagière de l'autre (Bessai, 2012) et les parlers font souvent l'objet de dévalorisation très accentuée. Cette vision de certains locuteurs à l'égard d'autres locuteurs ne partageant pas le même parler ou le même rapport aux langues est à l'origine de la glottophobie, car ces jugements stigmatisent généralement les pratiques vues comme déviantes de ce qui est considéré comme étant correct (Chachou, 2018). En effet, cette auto-catégorisation va dans le sens de la dévalorisation et de la dé-légitimation de l'autre en invoquant le critère linguistique (Chachou, 2019).

Ainsi, à la question du quartier où l'on parle le plus l'arabe bougiote et le kabyle, nous avons pu constater dans les réponses données un découpage spatio-linguistique de la ville pleinement assumé. Et nous avons ainsi constaté à travers les réponses données que le bougiote est toujours associé aux citadins de Bejaia et donc aux anciens quartiers de la ville comme: Karamane, Bab El Louz, etc., tandis que le kabyle est associé aux quartiers se trouvant en périphérie de la ville ou dans les nouveaux quartiers comme : Ihaddaden, Ighil Ouzoug, ou encore Tizi. La langue est ainsi vue comme un pôle d'identification (Gauthier, 2011), elle apparait comme une propriété toujours menacée aux yeux de certains locuteurs.

Ainsi, le fait d'associer sans gêne ni doute le bougiote à des quartiers bien précis de l'ancienne ville, et le kabyle aux nouveaux quartiers de la ville est une façon de renvoyer les individus à leur origine en passant par la langue. En d'autres termes, aux yeux de nos informateurs, ceux qui habitent l'ancienne ville parlent le bougiote et seraient ainsi les véritables citadins, tandis que les ruraux (ou les arrivistes) qui parlent bien évidement kabyle sont installés en périphérie ou dans les nouveaux quartiers de la ville (qui correspondent donc aux nouvelles constructions faites pour agrandir la ville et accueillir ses nouveaux arrivants).

Selon Chachou (2019), les critères que font prévaloir ces familles qui se disent originaires de la ville sont généralement la mise en avant des origines, et surtout de l'antériorité de l'occupation de la ville et, plus précisément, des anciens quartiers. Ainsi, ce découpage de la ville en deux (ancienne ville Vs nouvelle ville) reflète l'aspect conflictuel et glottophobe de ses habitants où d'un côté il y aurait " les vrais citadins » et donc les habitants légitimes, et de l'autre «les arrivistes» qui auraient donc beaucoup moins de légitimité à occuper l'espace.

Il semble que dans le cas présent, l'objectif de ces comportements glottophobes serait le contrôle social, où certains pensent qu'en tentant de réaliser une réduction de la pluralité linguistique, on garantit le contrôle sur les pratiques linguistiques, et donc un maintient du pouvoir. Et comme nous le confie Blanchet: «les pratiques linguistiques étant avant tout des pratiques sociales, le contrôle linguistique est un contrôle social » (2013 : 98), donc au-delà du caractère discriminatoire, il est aussi question de pouvoir. 


\section{Les représentations au service de la glottophobie}

Selon Taleb-Ibrahimi (1995) il existe un caractère généralement irréfléchi voire impulsif des représentations. Ainsi, l'idée que nos informateurs ont des « arrivistes » et l'idée qu'ils se font des langues et des choix discursifs de ces derniers ont un lien avec les représentations qui circulent dans le corps social. En effet, les représentations et les attitudes négatives qu'ils ont à l'égard de ceux qu'ils considèrent comme des intrus justifient, à leurs yeux, leur comportement glottophobe.

Nous avons pu constater que beaucoup de ces jeunes sont fortement influencés par leur milieu extérieur, et la vision qu'ils ont des pratiques discursives est fortement liée aux préjugés et aux représentations qui circulent dans leur environnement. Nous pouvons même dire que, dans le cas présent, l'individu et la société sont interdépendants au point que certaines représentations sont considérées comme des évidences. Ainsi, cette façon de considérer l'autre comme "arriviste " parce qu'il n'est pas natif et préfère parler en kabyle plutôt qu'en bougiote est vue comme allant de soi. Et le découpage linguistique de la ville fortement suggéré par les représentations en cours dans l'univers social ne pose problème à aucun d'entre eux.

Les représentations sont indissociables de l'individu et de ses comportements, et surtout des relations qu'il entretient avec l'autre, pour Médéric Gasquet-Cyrus : «les représentations sociales de la langue constituent un assemblage de savoirs permettant aux acteurs de co-construire une même réalité sociale, de s'assurer qu'ils la partagent, et d'adapter leurs comportements» (2009: 19). Ainsi, sans forcément se rendre compte, ces représentations fortement suggérées par l'entourage social donnent une certaine légitimité aux comportements glottophobes et autre stigmatisation des pratiques langagières en les justifiant. En somme, les représentations contribuent dans ce processus idéologico-discursif visant à hiérarchiser les langues et les pratiques langagières dans un but discriminatoire.

L'idée que ce parler serait plus approprié que tel autre parler, ou que telle langue serait plus juste que telle autre est guidée par les représentations en cours dans l'univers social, car le système des représentations est dépendant du contexte et de l'environnement où vit le sujet (Mohamed, 2003). Aussi, les représentations qu'ont ces jeunes bougiotes des "arrivistes" et de la légitimité à occuper certains espaces semblent donner une justification et une validité à leurs comportements glottophobes.

Cette étude a permis de cerner des traits spatio-discursifs caractéristiques de la ville de Bejaia. Nous avons pu constater que les comportements glottophobes pleinement assumés par les individus et ayant pour principal objectif la stigmatisation de l'autre servent à marginaliser et à isoler une partie de la ville. Notre enquête de terrain nous a permis de nous rendre compte à quel point la mutation de la ville liée à l'exode rural massif avait provoqué une fracture spatio-discursive entrainant un repli identitaire des anciennes familles bougiotes. En effet, le comportement glottophobe des bougiotes les pousse jusqu'à s'isoler de ceux qu'ils nomment « les arrivistes ", et ces deux groupes qui partagent un même territoire se côtoient mais gardent une certaine distance.

Le phénomène de glottophobie semble se répandre et se standardiser et donne à voir une nouvelle forme de routines langagières dans la ville où la stigmatisation est banalisée. Ces comportements glottophobes tracent les frontières sociales et sont devenus des indicateurs des relations qui opposent les occupants de ce même espace. 
Il est évident que les représentations, le rapport à l'espace, et l'attachement à la langue maternelle ont fini par faire naître des discriminations linguistiques où certains parlers ou variétés linguistiques sont perçus comme inférieurs et où les individus qui les parlent se retrouvent automatiquement stigmatisés. Nous pouvons ainsi dire que, la discrimination langagière a toujours un impact relationnel sur les occupants d'un même espace, toutefois cet impact est différent selon que cette ségrégation soit subie ou infligée. Aussi, il serait intéressant de voir comment cette glottophobie est vécue par ceux qu'on nomme « les arrivistes».

\section{BIBLIOGRAPHIE}

Arditty, J., Blanchet, P., 2008, « La « mauvaise langue » des « ghettos linguistiques » : la glottophobie française, une xénophobie qui s'ignore », Revue Asylons $n^{\circ} 3 \mid$ Institutionnalisation de la xénophobie. Disponible sur : [http://www.reseau-terra.eu/article748.html], (consulté le 19/10/2019).

Aoumer, F., 2009, Renversement de situation : l'arabe de Bougie, un très ancien parler arabe citadin menacé par le berbère, Revue des Études Berbères. Disponible sur : [http:// centrederechercheberbere.fr/renversement-de-situation-larabe-de-bougie-un-tres-ancienparler-arabe-citadin-menace-par-le-berbere.html], (consulté le : 01/12/2019)

Arezki, A., 2008, «L'identité linguistique : une construction sociale et/ou un processus de construction socio-discursive ", Synergies Algérie, n² 2, pp. 191-198.

Benbelaid, L., 2014, Les parlers jeunes de la ville de Bejaia entre identité et représentation (la glocalisation des pratiques langagières dites culturelles des jeunes), thèse de doctorat.

Bessai, B., 2012, «Plurilinguisme et insécurité linguistique en Algérie : Paroles de lycéen (ne)s à Bejaïa ", Corela [En ligne], 10-2 | 2012, mis en ligne le 10 décembre 2012, Disponible sur : [http:// corela.revues.org/2762; DOI : 10.4000/corela.2762], (consulté le : 01/10/2016).

Boutet, J., 1997, Langage et société, Paris, le Seuil.

Bulot, T., 2004, «Les frontières et territoires intra-urbains : évaluation des pratiques et discours épilinguistiques ", in Bombi R., Fusco F., Città plurilingui. Lingue e culture a confronto in situazioni urbane / Multilingual cities, Actes du colloque international, Udine, 5-7 décembre 2002, Udine, Forum, p. 110-125.

Bulot, T., 2008, « Normes et identités en rupture : la fragmentation des espaces », dans Mehrsprachigkeit in frankophonen Räumen, Martin Meidenbauer Verlag, München, p. 11-25.

Bulot, T., 2013, « Discrimination sociolinguistique et pluralité des normes identitaires.

Linguicisme de référence et linguicisme d'action », Cahiers internationaux de sociolinguistique, vol. 4, n 2, p. 7-27.

Blanchet, P., 2013, « Repères terminologiques et conceptuels pour identifier les discriminations linguistiques ", Cahiers internationaux de sociolinguistique, $n^{\circ}$ 4, p. 29-39.

Blanchet, P., 2016, Discriminations : combattre la glottophobie, Paris, Textuel. 
Chachou, I., 2009, « Remarques sur le parler urbain de Mostaganem », Synergies Algérie, Contacts et diversités linguistiques, $\mathrm{n}^{\circ} 4$, p. 69-81.

Chachou, I., 2012, «L'auto-désignation et l'hétéro-désignation comme procédés langagiers de ségrégation urbaine : le cas de la ville algérienne de Mostaganem », Revue Synergies Algérie, $n^{\circ} 15$, p. 169-177.

Chachou, I., 2018, « "Les gens qui me reprochent mon accent, je ne m'appelle pas Marianne, je suis Algérienne" : Les perceptions de la norme du français pratiqué en Algérie ", Revue Signe, Discours et Société $n^{\circ} 19$, Normes, fictions, pratiques langagières : l'imaginaire linguistique.

Chachou, I., 2019, « « Mostaganem avant l'envahissement » : fragments de discours numériques autour de l'appropriation de l'espace de la ville », Les Cahiers d'EMAM [En ligne], 31 | 2019, mis en ligne le 25 septembre 2019. Disponible sur : [http://journals.openedition.org/emam/2128], (Consulté le 27/09/2019).

Calvet, L-J., 1994, Les Voix de la ville : introduction à la sociolinguistique urbaine. Paris, Payot.

De Habsbourg, L-S., 1999, Bougie, La perle de l'Afrique du Nord, l'Harmattan.

Gasquet-Cyrus M., Petitjean, C. (Dir.), 2009, Le poids des langues : dynamiques, représentations, contacts, conflits, Paris, l'Harmattan.

Gauthier, C., 2011, Changer de langue pour échapper à la langue ? L' "identité linguistique" en question, Revue de littérature comparée, $\mathrm{n}^{\circ} 338$, p. 183-196. Disponible sur : [https:// www.cairn.info/revue-de-litterature-comparee-2011-2-page-183.htm], (consulté le 12/05/2020)

Lasagabaster, D., 2006, « Les attitudes linguistiques : un état des lieux », Revue Éla. Études de linguistique appliquée, $\mathrm{n}^{\circ}$ 144, p. 393-406. Disponible sur : [http:// www.cairn.info/revueela-2006-4-page-393.htm], (consulté le 15/06/2020)

Messaoudi, L., 2001, « Urbanisation linguistique et dynamique langagière dans la ville de Rabat », Cahiers de sociolinguistique, vol. $6, \mathrm{n}^{\circ} 1$, p. 89-100.

Mohamed, A., 2003, « Langues et identité. Les jeunes maghrébins de l'immigration », Revue européenne des migrations internationales, vol. 20, n³, pp. 196-197.

Taleb-Ibrahimi, K., 1995, Les Algériens et leur(s) langue(s), Alger, El Hikma.

Thiébault, J-Y., 2006, Thèmes culturels, Paris, Vuibert.

\section{NOTES}

1. Qui tend même à surpasser l'arabe bougiote.

2. Une façon de les renvoyer à leur origine rurale.

3. Ou nouvelle ville.

4. Désignations visant à décrire une appartenance à des groupes sociaux à l'intérieur de la ville, elles passent par la définition de soi, de soi par rapport à l'autre, et de l'autre par rapport à soi. Pour Chachou (2012), Ces attributs stigmatisants servent aux groupes d'une même ville à se démarquer les uns des autres et à s'affirmer les uns par rapport aux autres. 


\section{RÉSUMÉS}

Nous nous intéressons ici aux comportements glottophobes des citadins de Bejaia à l'égard des nouveaux arrivants. Cette glottophobie reflète un nouveau rapport de l'individu à la langue et révèle les tensions présentes entre les habitants de la ville. À travers une approche sociolinguistique, nous avons tenté de comprendre ce phénomène et d'en mesurer l'étendue. En effet, notre enquête de terrain nous a permis de découvrir un profond malaise entre les habitants de la ville, et une grande fracture intra-urbaine. Il ressort de cette étude des comportements glottophobes pleinement assumés et justifiés par une légitimité à occuper l'espace.

We are interested here in the glottophobic behaviors of the townspeople of Bejaia towards the newcomers. This glottophobia reflects a new relationship of the individual to the language and reveals the tensions between the city's inhabitants. Through a sociolinguistic approach, we tried to understand this phenomenon and measure its extent. Indeed, our field survey allowed us to discover a deep discomfort between the inhabitants of the city, and a great intra-urban divide. This study shows glottophobic behaviors fully assumed and justified by a legitimacy to occupy space.

INDEX

Keywords : glottophobia, self-categorization, identity, representation, sociolinguistics

Mots-clés : glottophobie, auto-catégorisation, identité, représentation, sociolinguistique

\section{AUTEUR}

\section{LYDIA BENBELAID}

Université de Bejaia, Algérie 